\title{
A new role for histone tail modifications in transcription elongation
}

\author{
Grant A. Hartzog and John W. Tamkun ${ }^{1}$ \\ Department of Molecular, Cell, and Developmental Biology, University of California at Santa Cruz, Santa Cruz, California \\ 95064, USA
}

RNA polymerase II (Pol II) faces a number of daunting tasks while transcribing genes. It must respond to a large array of transcription regulators, permitting distinct regulation of thousands of genes. It must coordinate its activities with RNA processing events and regulators of chromatin modifications and structure (Sims et al. 2004; Saunders et al. 2006). The C-terminal domain (CTD), a unique structure at the $\mathrm{C}$ terminus of Pol II's largest subunit, permits coordination of these activities (Phatnani and Greenleaf 2006). The CTD consists of 26 (in yeast) to 52 (in mice and humans) copies of a heptapeptide motif, $\mathrm{Y}_{1} \mathrm{~S}_{2} \mathrm{P}_{3} \mathrm{~T}_{4} \mathrm{~S}_{5} \mathrm{P}_{6} \mathrm{~S}_{7}$, and is subject to phosphorylation and dephosphorylation during transcription. These modifications serve as spatial and temporal markers of Pol II's progress through a cycle of gene transcription.

\section{Coupling of CTD modifications to histone modifications and RNA processing}

The CTD serves as a phosphorylation-regulated scaffold that recruits distinct sets of transcription regulators, RNA processing factors, and chromatin modifiers during the different phases of the transcription cycle (Phatnani and Greenleaf 2006). This strategy allows transcription and RNA processing factors to be recruited to their sites of actions; it also helps to lay down the patterns of histone modifications that are thought to help regulate transcription and maintain zones of distinct chromatin structure and dynamics across promoters and transcribed regions of genes (Li et al. 2007). Prior to initiation, the hypophosphorylated CTD binds the Mediator complex, which facilitates communication between Pol II and promoter-bound transcription regulators. Around the time of initiation, the CTD is hyperphosphorylated at Ser5, triggering release of the Mediator and recruitment of the capping enzyme and a histone H3 Lys4 (H3K4) methyltransferase. This insures that RNA transcripts will be capped early in the transcription cycle and helps to explain the polarity of $\mathrm{H} 3 \mathrm{~K} 4$ methylation ob-

${ }^{1}$ Corresponding author.

E-MAIL tamkun@biology.ucsc.edu; FAX (831) 459-3139.

Article is online at http://www.genesdev.org/cgi/doi/10.1101/gad.1628707. served on active genes, with trimethylation predominating at the 5' end, giving way to di- and monomethylation as one moves toward the $3^{\prime}$ end of the gene. As Pol II moves further from the promoter, Ser5 phosphorylation levels begin to drop and levels of Ser2 phosphorylation increase. In yeast, the doubly phosphorylated, phosphoSer2,5 form of the CTD binds Set2, a histone H3K36 methyltransferase, explaining the localization of this chromatin mark to transcribed regions. Binding specificity of polyadenylation factors for phospho-Ser2 or phospho-Ser2,5 forms of the CTD may similarly explain their localization at the 3 ' ends of genes (Phatnani and Greenleaf 2006).

\section{Paused polymerases}

In addition to the cycle of CTD modification, Pol II's behavior changes in another important way as it moves down a gene. In at least some, and perhaps many cases, Pol II pauses shortly after initiating transcription, 25-50 nucleotides downstream from the start-site. These polymerases are transcriptionally engaged; they are associated with a nascent transcript and have an open transcription bubble. This phenomenon was first observed in Drosophila at heat-shock genes and later at several cellular oncogenes (Saunders et al. 2006). In addition to repressing or attenuating transcription, polymerase pausing permits a gene to be poised for rapid activation in response to environmental or developmental signals.

More recently, genome-wide chromatin immunoprecipitation (ChIP) studies demonstrated that Pol II is near the $5^{\prime}$ ends of many genes, including inactive genes (Kim et al. 2005; Barski et al. 2007; Guenther et al. 2007). Nucleosomes at the 5' ends of many of these inactive, but polymerase-associated, genes have post-translational modifications normally associated with active transcription: trimethylation of $\mathrm{H} 3 \mathrm{~K} 4$ and acetylation of $\mathrm{H} 3 \mathrm{~K} 9$ and K14 (Kim et al. 2005; Barski et al. 2007; Guenther et al. 2007). In addition, RNA transcripts derived from the $5^{\prime}$, but not $3^{\prime}$, end of these genes can be detected (Guenther et al. 2007). Thus, a large fraction of all genes are associated with short transcripts derived from their 5' ends as well as with chromatin marks of initiation, but 
not elongation. Whether this is indicative of widespread paused polymerases or of some other phenomenon remains to be determined (Guenther et al. 2007). However, these findings suggest that the regulation of early stages of transcriptional elongation may be a relatively common phenomenon in higher eukaryotes.

\section{Regulation of pausing}

Support for the idea that early elongation is subject to regulation comes from the pioneering studies of Price and colleagues (Peterlin and Price 2006), who found that early elongation by Pol II is subject to negative regulation and that a Ser2-specific CTD kinase, positive transcription elongation factor $\mathrm{b}(\mathrm{P}-\mathrm{TEFb})$, is required to overcome this block to elongation. Later work suggested that the DRB sensitivity-inducing factor (DSIF) and negative elongation factor (NELF) complexes may be responsible for this negative regulation, and that $\mathrm{P}-\mathrm{TEFb}$ may target these complexes in addition to Pol II (Peterlin and Price 2006; Saunders et al. 2006). P-TEFb activity stimulates release of NELF from elongation complexes, releases Pol II from the early block to elongation, and converts DSIF into a positive regulator of elongation (Saunders et al. 2006).

P-TEFb has at least two important effects on gene expression; it stimulates Pol II processivity-i.e., the probability that an elongating polymerase will reach the end of a gene-and it promotes appropriate pre-mRNA processing via its phosphorylation of Ser2 of the CTD repeat (Peterlin and Price 2006; Li et al. 2007). Studies in which $\mathrm{P}-\mathrm{TEFb}$ was inhibited with flavopiridol have come to different conclusions on P-TEFb's role in elongation in vivo. For example, in one study, the inhibition of $\mathrm{P}-\mathrm{TEFb}$ dramatically reduced Pol II transcription /Chao and Price 2001). In a second study, the density of Pol II over a gene was not significantly altered by flavopiridol treatment, leading to the suggestion that P-TEFb's primary role in gene expression may be to coordinate transcription with 3 ' end processing (Ni et al. 2004). One possibility is that the role of $\mathrm{P}-\mathrm{TEFb}$ depends in part on whether or not a gene has a paused polymerase. Definitive identification of genes with paused polymerases may aid in resolution of this issue. A second possibility is that some other attribute of a gene, such as its length or the density of introns, will determine the nature of its P-TEFb dependence.

An intriguing open question is whether the regulation of early elongation is used to modulate transcription in a gene-specific manner or in response to particular stimuli. Several observations suggest that P-TEFb activity and early elongation can be regulated in a gene-specific manner. First, P-TEFb functions as a strong activator of transcription when tethered to a promoter (Lis et al. 2000; Raha et al. 2005). Second, there is evidence that a subset of promoter-bound transcription activators can recruit P-TEFb to specific genes (Saunders et al. 2006). Third, the HIV Tat protein recruits P-TEFb to the HIV LTR and stimulates processive elongation of Pol II across the HIV genome (Peterlin and Price 2006).

\section{H3S10 phosphorylation is associated with actively transcribed regions of chromatin in nondividing cells}

Given the central role of P-TEFb in early elongation, there is considerable interest in the mechanisms that regulate its recruitment and activity. A study by Ivaldi et al. (2007) in a recent issue of Genes \& Development presents evidence that a covalent modification of nucleosomal histones-the phosphorylation of Ser10 of histone $\mathrm{H} 3(\mathrm{H} 3 \mathrm{~S} 10)$ - plays a key role in this process in Drosophila.

H3S10 phosphorylation has been implicated in chromosome condensation and segregation, but a growing body of evidence suggests that this modification also plays an important role in transcriptional activation (Nowak and Corces 2004). More than 20 years ago, H3S10 phosphorylation was found to accompany the activation of immediate early response genes (including c-fos and c-jun) in response to mitogenic signals. H3S10 phosphorylation was subsequently shown to accompany the activation of numerous other genes in organisms ranging from yeast to humans. The changes in the chromosomal distribution of H3S10 phosphorylation are particularly dramatic during the heat-shock response in Drosophila (Nowak and Corces 2000). When larvae are exposed to elevated temperatures, the transcription of heat-shock genes is rapidly activated as other genes become transcriptionally silent. These genome-wide changes in gene expression are mirrored by a redistribution of H3S10 phosphorylation to heat-shock genes, including the Hsp70 gene cluster. These observations suggested that H3S10 phosphorylation might play a relatively global role in transcription by Pol II.

\section{The JIL-1 histone kinase is required for the activation of Drosophila heat-shock genes}

Numerous H3S10 kinases have been identified. Mitotic H3S10 kinases include AuroraB and other members of the Aurora/Ipl 1 kinase family; H3S10 kinases implicated in transcriptional regulation include the yeast Snf1 and mammalian Pim1, Rsk2, Msk1, and Msk2 kinases (Nowak and Corces 2004; Johansen and Johansen 2006). In Drosophila, the JIL-1 kinase is responsible for the majority of H3S10 phosphorylation in interphase cells (Jin et al. 1999; Wang et al. 2001). To determine whether H3S10 phosphorylation plays a causal role in transcription, Ivaldi et al. (2007) examined the role of JIL-1 in the heat-shock response. JIL-1 is associated with the majority of transcriptionally active genes, as evidenced by the extensive overlap between the distributions of JIL-1 and Pol II on polytene chromosomes. Prior to heat shock, little or no JIL-1 is present at the transcriptionally silent heat-shock genes. Following heat shock, JIL-1 levels rapidly increase in the vicinity of the heat-shock genes as their transcription is activated. Consistent with an important role for H3S10 phosphorylation in gene expression, the activation of $H s p 70$ transcription in response to heat shock is blocked in $J I L-1$ mutant larvae (Ivaldi et al. 2007). 
JIL-1 is required for the recruitment of the P-TEFb kinase to promoters

Ivaldi et al. (2007) next investigated which stage of the transcription cycle is dependent on JIL-1. The loss of JIL-1 function has no effect on the binding of HSF to heat-shock genes following heat shock, suggesting that H3S10 phosphorylation acts downstream from transcription factor recruitment. The level of Pol IIo ${ }^{\text {ser5 }}$ associated with heat-shock genes is also normal in $J I L-1$ mutants, suggesting that H3S10 phosphorylation is not required for either transcriptional initiation or promoter clearance. By contrast, the loss of JIL-1 function leads to a dramatic reduction in the levels of both the CycT subunit of $\mathrm{P}-\mathrm{TEFb}$ and Pol $\mathrm{IIo}^{\text {ser2 }}$ associated with Hsp70 genes. The simplest interpretation of these findings is that H3S10 phosphorylation stimulates transcription by recruiting the P-TEFb kinase to promoters.

How does the JIL-1-dependent recruitment of P-TEFb stimulate $H s p 70$ transcription? One plausible model is that the phosphorylation of DSIF, NELF, and/or Ser2 of the CTD by P-TEFb is required to relieve promoterproximal pausing at $H s p 70$ (and perhaps other) genes. As noted by Ivaldi et al. (2007), an alternative possibility is that elongation proceeds normally in $J I L-1$ mutants, but the failure to phosphorylate Ser2 of the CTD disrupts the recruitment of factors that process the nascent $H s p 70$ mRNA, leading to its rapid degradation.

\section{H3S10 phosphorylation plays a relatively global role in transcription by Pol II in Drosophila}

Is $\mathrm{H} 3 \mathrm{~S} 10$ phosphorylation required for the transcription of non-heat-shock genes? There is a striking correlation between the genome-wide distributions of JIL-1, H3S10 phosphorylation, and Pol II on Drosophila polytene chromosomes (Nowak and Corces 2000; Ivaldi et al. 2007), and the overall level of Pol $\mathrm{II}^{\text {ser2 }}$, but not Pol $\mathrm{IIo}^{\text {ser5 }}$, is significantly reduced in $J I L-1$ mutant larvae even prior to heat shock (Ivaldi et al. 2007). Additional evidence that H3S10 phosphorylation plays a global role in transcription by Pol II has been provided by studies of the Drosophila serine/threonine protein phosphatase 2A (PP2A). The inactivation of non-heat-shock genes at elevated temperatures is accompanied by the loss of H3S10 phosphorylation; loss of PP2A function blocks this dephosphorylation as well as the global repression of non-heatshock genes (Nowak et al. 2003). These observations suggest that H3S10 phosphorylation mediated by JIL-1 may be required for transcription of most, if not all, Drosophila genes transcribed by Pol II.

\section{Targeting and regulation of JIL-1 kinase activity}

Relatively little is known about the mechanisms that regulate the recruitment and activity of the JIL-1 kinase to its target genes. The recruitment of H3S10 kinases by transcription factors is well documented in other organisms (Lo et al. 2005; Zippo et al. 2007), and a similar mechanism is likely to recruit JIL-1 to its target genes in
Drosophila. As discussed above, genetic studies have suggested that the PP2A phosphatase negatively regulates JIL-1 function in vivo (Nowak et al. 2003). By counteracting JIL-1-mediated H3S10 phosphorylation, PP2A may function as a negative regulator of $\mathrm{P}-\mathrm{TEFb}$ recruitment and transcriptional elongation.

\section{How does H3S10 phosphorylation modulate chromatin structure and transcription?}

Much remains to be learned about how JIL-1 and other H3S10 kinases modulate chromatin structure and transcription. JIL-1 plays an important role in the regulation of higher-order chromatin structure, as evidenced by the striking polytene chromosome defects observed in $J I L-1$ mutant larvae (Deng et al. 2005). However, JIL-1 is not required for the formation of heat-shock puffs or the recruitment of HSF and Pol II to heat-shock genes following their activation, suggesting that H3S10 phosphorylation does not promote transcription merely by creating an open chromatin configuration in which DNA is more accessible to the transcriptional machinery (Ivaldi et al. 2007). Another popular hypothesis is that the phosphorylation of H3S10 modulates transcription by influencing other covalent modifications of the histone $\mathrm{H} 3$ tail, including the methylation of Lys9 and the acetylation of Lys 14 (Nowak and Corces 2004; Johansen and Johansen 2006). Consistent with this view, JIL-1 helps maintain the distinction between euchromatic and heterochromatic domains by preventing ectopic $\mathrm{H} 3 \mathrm{~K} 9$ methylation and HP1 binding (Zhang et al. 2006). Studies of the Snf1 kinase in budding yeast have shown that the phosphorylation of H3S10 can activate transcription by promoting the acetylation of Lys14 of the $\mathrm{H} 3$ tail by the Gen 5 histone acetyltransferase (Lo et al. 2001). It is also possible that H3S10 phosphorylation directly regulates the interaction of structural or regulatory proteins with the histone $\mathrm{H} 3$ tail. For example, a recent study suggests that H3S10 phosphorylation may block binding of the RSC chromatin remodeler to acetylated histone tails (VanDemark et al. 2007). Relatively few effectors of H3S10 phosphorylation have been identified, but recent studies have shown that mammalian 14-3-3 proteins directly bind histone $\mathrm{H} 3$ tails phosphorylated on Ser10 in vitro and are recruited to nucleosomes at the c-fos and c-jun promoters following their activation in vivo (Macdonald et al. 2005). Finally, it remains possible that JIL-1 regulates chromatin structure and gene expression by phosphorylating proteins other than histone H3. It remains to be seen which, if any, of the above mechanisms are involved in the JIL-1-mediated recruitment of P-TEFb in Drosophila.

\section{Does dosage compensation involve changes in early elongation?}

The findings of Ivaldi et al. (2007) may also provide a molecular explanation for the involvement of JIL-1 in dosage compensation, a process that has fascinated mo- 
lecular and developmental biologists for many years. In Drosophila, dosage compensation is achieved via a twofold increase in the transcription of X-linked genes in males. This process is dependent on the dosage compensation complex, which recruits the Males absent on the first (MOF) histone acetyltransferase to the male X chromosome, causing the widespread acetylation of Lys16 of histone H4 (H4K16) (Straub and Becker 2007). JIL-1 physically interacts with the dosage compensation complex, leading to the increased phosphorylation of H3S10 on the male X chromosome (Jin et al. 2000; Wang et al. 2001). The elevated phosphorylation of H3S10 on the male $\mathrm{X}$ appears to contribute to the increased transcription of at least some X-linked genes in males, since the partial loss of JIL-1 function can interfere with dosage compensation and has a disproportionate effect on male viability (Wang et al. 2001; Lerach et al. 2006). Thus, dosage compensation involves two covalent modifications of chromatin: H4K16 acetylation (which is restricted to the male X chromosome) and H3S10 phosphorylation (which is elevated on the male X chromosome). H4K16 acetylation is thought to disrupt interactions between nucleosomes or antagonize chromatin compaction mediated by other proteins (Rea et al. 2007). How does H3S10 phosphorylation contribute to dosage compensation? Based on the findings of Ivaldi et al. (2007), it is tempting to speculate that the elevated phosphorylation of H3S10 might contribute to dosage compensation by stimulating P-TEFb recruitment and early elongation. Further work will be necessary to test this hypothesis and clarify the relative contributions of H4K16 acetylation and H3S10 phosphorylation to dosage compensation.

\section{Is $\mathrm{H} 3 \mathrm{~S} 10$ phosphorylation a gene-specific regulator of transcription?}

The work of Ivaldi et al. (2007) highlights the possibility that $\mathrm{P}-\mathrm{TEFb}$ recruitment to elongation complexes can be used to flexibly and broadly regulate transcription elongation and its linkages to RNA processing and chromatin. As noted above, P-TEFb can be recruited to genes via multiple mechanisms. JIL-1 is not the only H3S10 kinase, and H3S10 can be dephosphorylated by PP2A, suggesting that a balance of kinases and phosphatases, some of which may be targeted in a gene-specific manner, regulate $\mathrm{P}-\mathrm{TEFb}$ localization on genes.

It is not clear that the role of H3S10 phosphorylation in P-TEFb recruitment is conserved in all eukaryotes. In budding yeast, H3S10 phosphorylation appears to facilitate a relatively early step in transcription at the INO1 gene, including the recruitment of the TATA-binding protein to specific promoters (Lo et al. 2005). However, H3S10 mutations do not cause significant growth defects, arguing against a global role in transcription for this modification in yeast (Hsu et al. 2000). In mammals, H3S10 phosphorylation has been implicated in the transcription of many, but not all, inducible genes (Nowak and Corces 2004; Johansen and Johansen 2006). A recent study of a human H3S10 kinase, PIM1, suggests that this modification may play an important role in the transcription of a relatively large number of genes (Zippo et al. 2007). MYC recruits PIM1 to at least two of its target genes, FOSL1 and ID2, leading to the phosphorylation of H3S10 in the vicinity of MYC-binding sites. PIM1 is required for the activation of both FOSL1 and ID2 by MYC, and gene expression profiling revealed that this $\mathrm{H} 3 \mathrm{~S} 10$ kinase is also required for the regulated expression of $>200$ other MYC targets. Interestingly, PIM1 knock-down has a disproportionately strong effect on the levels of Pol IIo ${ }^{\text {ser2 }}$ associated with FOSL1 and ID2, as would be expected for a factor that stimulates P-TEFb recruitment or activity. Additional information about the genome-wide distribution of H3S10 phosphorylation and its effect on specific stages of the transcription cycle should clarify the role of this histone modification in gene expression in mammals.

Brd4, a tandem bromodomain protein that interacts with acetylated histones, appears to also recruit $\mathrm{P}-\mathrm{TEFb}$ to promoters in mammals (Jang et al. 2005; Yang et al. 2005), suggesting an additional connection between chromatin marks characteristic of active chromatin and $\mathrm{P}-\mathrm{TEFb}$ recruitment. P-TEFb may also regulate factors that lay down chromatin modifications over transcribed regions. One of these modifications, methylation of H3K36, antagonizes inappropriate histone acetylation events over the body of genes (Carrozza et al. 2005). One intriguing idea is that the interplay between histone modifications and P-TEFb activity serves to create or sharpen boundaries of functionally distinct domains (i.e., early vs. late elongation) in transcription units. Comparison of the distribution of P-TEFb, H3S10 phosphorylation, JIL-1, and chromatin marks of early and late elongation may be particularly informative in this regard.

\section{Future directions}

This work raises a number of important questions. Do other H3S10 kinases, including PIM1, regulate P-TEFb recruitment? How does $\mathrm{H} 3 \mathrm{~S} 10$ phosphorylation regulate P-TEFb recruitment? Are 14-3-3 proteins the only effectors of H3S10 phosphorylation, or does this modification influence the interaction of other structural or regulatory proteins with chromatin? With the growing realization that paused polymerases are relatively common in eukaryotic genomes, JIL-1 and other factors that regulate $\mathrm{P}-\mathrm{TEFb}$ function will continue to be of great interest to molecular biologists.

\section{Acknowledgments}

Work in our laboratories is supported by grants from the National Institutes of Health.

\section{References}

Barski, A., Cuddapah, S., Cui, K., Roh, T.Y., Schones, D.E., Wang, Z., Wei, G., Chepelev, I., and Zhao, K. 2007. Highresolution profiling of histone methylations in the human genome. Cell 129: 823-837. 
Carrozza, M.J., Li, B., Florens, L., Suganuma, T., Swanson, S.K., Lee, K.K., Shia, W.J., Anderson, S., Yates, J., Washburn, M.P., et al. 2005. Histone H3 methylation by Set2 directs deacetylation of coding regions by Rpd3S to suppress spurious intragenic transcription. Cell 123: 581-592.

Chao, S.H. and Price, D.H. 2001. Flavopiridol inactivates $\mathrm{P}-\mathrm{TEFb}$ and blocks most RNA polymerase II transcription in vivo. J. Biol. Chem. 276: 31793-31799.

Deng, H., Zhang, W., Bao, X., Martin, J.N., Girton, J., Johansen, J., and Johansen, K.M. 2005. The JIL-1 kinase regulates the structure of Drosophila polytene chromosomes. Chromosoma 114: 173-182.

Guenther, M.G., Levine, S.S., Boyer, L.A., Jaenisch, R., and Young, R.A. 2007. A chromatin landmark and transcription initiation at most promoters in human cells. Cell 130: $77-$ 88

Hsu, J.Y., Sun, Z.W., Li, X., Reuben, M., Tatchell, K., Bishop, D.K., Grushcow, J.M., Brame, C.J., Caldwell, J.A., Hunt, D.F., et al. 2000. Mitotic phosphorylation of histone H3 is governed by Ipl1/aurora kinase and Glc7/PP1 phosphatase in budding yeast and nematodes. Cell 102: 279-291.

Ivaldi, M.S., Karam, C.S., and Corces, V.G. 2007. Phosphorylation of histone $\mathrm{H} 3$ at Ser10 facilitates RNA polymerase II release from promoter-proximal pausing in Drosophila. Genes Dev. 21: 2818-2381.

Jang, M.K., Mochizuki, K., Zhou, M., Jeong, H.S., Brady, J.N., and Ozato, K. 2005. The bromodomain protein Brd4 is a positive regulatory component of P-TEFb and stimulates RNA polymerase II-dependent transcription. Mol. Cell 19: 523-534.

Jin, Y., Wang, Y., Walker, D.L., Dong, H., Conley, C., Johansen, J., and Johansen, K.M. 1999. JIL-1: A novel chromosomal tandem kinase implicated in transcriptional regulation in Drosophila. Mol. Cell 4: 129-135.

Jin, Y., Wang, Y., Johansen, J., and Johansen, K.M. 2000. JIL-1, a chromosomal kinase implicated in regulation of chromatin structure, associates with the male specific lethal (MSL) dosage compensation complex. J. Cell Biol. 149: 1005-1010.

Johansen, K.M. and Johansen, J. 2006. Regulation of chromatin structure by histone H3S10 phosphorylation. Chromosome Res. 14: 393-404.

Kim, T.H., Barrera, L.O., Zheng, M., Qu, C., Singer, M.A., Richmond, T.A., Wu, Y., Green, R.D., and Ren, B. 2005. A highresolution map of active promoters in the human genome. Nature 436: 876-880.

Lerach, S., Zhang, W., Bao, X., Deng, H., Girton, J., Johansen, J., and Johansen, K.M. 2006. Loss-of-function alleles of the JIL-1 kinase are strong suppressors of position effect variegation of the wm4 allele in Drosophila. Genetics 173: 2403-2406.

Li, B., Carey, M., and Workman, J.L. 2007. The role of chromatin during transcription. Cell 128: 707-719.

Lis, J.T., Mason, P., Peng, J., Price, D.H., and Werner, J. 2000. $\mathrm{P}-\mathrm{TEFb}$ kinase recruitment and function at heat shock loci. Genes \& Dev. 14: 792-803.

Lo, W.S., Duggan, L., Emre, N.C., Belotserkovskya, R., Lane, W.S., Shiekhattar, R., and Berger, S.L. 2001. Snf1-A histone kinase that works in concert with the histone acetyltransferase Gcn5 to regulate transcription. Science 293: 11421146.

Lo, W.S., Gamache, E.R., Henry, K.W., Yang, D., Pillus, L., and Berger, S.L. 2005. Histone H3 phosphorylation can promote TBP recruitment through distinct promoter-specific mechanisms. EMBO J. 24: 997-1008.

Macdonald, N., Welburn, J.P., Noble, M.E., Nguyen, A., Yaffe, M.B., Clynes, D., Moggs, J.G., Orphanides, G., Thomson, S., Edmunds, J.W., et al. 2005. Molecular basis for the recogni- tion of phosphorylated and phosphoacetylated histone h3 by 14-3-3. Mol. Cell 20: 199-211.

Ni, Z., Schwartz, B.E., Werner, J., Suarez, J.R., and Lis, J.T. 2004. Coordination of transcription, RNA processing, and surveillance by P-TEFb kinase on heat shock genes. Mol. Cell 13: 55-65.

Nowak, S.J. and Corces, V.G. 2000. Phosphorylation of histone H3 correlates with transcriptionally active loci. Genes \& Dev. 14: 3003-3013.

Nowak, S.J. and Corces, V.G. 2004. Phosphorylation of histone H3: A balancing act between chromosome condensation and transcriptional activation. Trends Genet. 20: 214-220.

Nowak, S.J., Pai, C.Y., and Corces, V.G. 2003. Protein phosphatase $2 \mathrm{~A}$ activity affects histone $\mathrm{H} 3$ phosphorylation and transcription in Drosophila melanogaster. Mol. Cell. Biol. 23: 6129-6138.

Peterlin, B.M. and Price, D.H. 2006. Controlling the elongation phase of transcription with P-TEFb. Mol. Cell 23: 297-305.

Phatnani, H.P. and Greenleaf, A.L. 2006. Phosphorylation and functions of the RNA polymerase II CTD. Genes \& Dev. 20: 2922-2936.

Raha, T., Cheng, S.W., and Green, M.R. 2005. HIV-1 Tat stimulates transcription complex assembly through recruitment of TBP in the absence of TAFs. PLOS Biol. 3: e44. doi: 10.1371/journal.pbio.0030044.

Rea, S., Xouri, G., and Akhtar, A. 2007. Males absent on the first (MOF): From flies to humans. Oncogene 26: 5385-5394.

Saunders, A., Core, L.J., and Lis, J.T. 2006. Breaking barriers to transcription elongation. Nat. Rev. Mol. Cell Biol. 7: 557567.

Sims III, R.J., Belotserkovskaya, R., and Reinberg, D. 2004. Elongation by RNA polymerase II: The short and long of it. Genes \& Dev. 18: 2437-2468.

Straub, T. and Becker, P.B. 2007. Dosage compensation: The beginning and end of generalization. Nat. Rev. Genet. 8: $47-$ 57.

VanDemark, A.P., Kasten, M.M., Ferris, E., Heroux, A., Hill, C.P., and Cairns, B.R. 2007. Autoregulation of the Rsc4 tandem bromodomain by Gen5 acetylation. Mol. Cell 27: $817-$ 828 .

Wang, Y., Zhang, W., Jin, Y., Johansen, J., and Johansen, K.M. 2001. The JIL-1 tandem kinase mediates histone H3 phosphorylation and is required for maintenance of chromatin structure in Drosophila. Cell 105: 433-443.

Yang, Z., Yik, J.H., Chen, R., He, N., Jang, M.K., Ozato, K., and Zhou, Q. 2005. Recruitment of P-TEFb for stimulation of transcriptional elongation by the bromodomain protein Brd4. Mol. Cell 19: 535-545.

Zhang, W., Deng, H., Bao, X., Lerach, S., Girton, J., Johansen, J., and Johansen, K.M. 2006. The JIL-1 histone H3S10 kinase regulates dimethyl H3K9 modifications and heterochromatic spreading in Drosophila. Development 133: 229-235.

Zippo, A., De Robertis, A., Serafini, R., and Oliviero, S. 2007. PIM1-dependent phosphorylation of histone $\mathrm{H} 3$ at serine 10 is required for MYC-dependent transcriptional activation and oncogenic transformation. Nat. Cell Biol. 9: 932-944. 


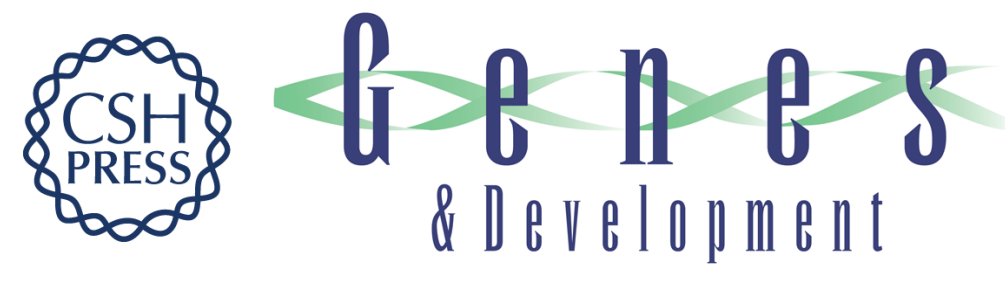

\section{A new role for histone tail modifications in transcription elongation}

Grant A. Hartzog and John W. Tamkun

Genes Dev. 2007, 21:

Access the most recent version at doi:10.1101/gad.1628707

\section{Related Content Phosphorylation of histone H3 at Ser10 facilitates RNA polymerase II release from promoter-proximal pausing in Drosophila \\ M. Soledad Ivaldi, Caline S. Karam and Victor G. Corces \\ Genes Dev. November , 2007 21: 2818-2831}

References This article cites 34 articles, 12 of which can be accessed free at:

http://genesdev.cshlp.org/content/21/24/3209.full.html\#ref-list-1

Articles cited in:

http://genesdev.cshlp.org/content/21/24/3209.full.html\#related-urls

\section{License}

Email Alerting

Service

Receive free email alerts when new articles cite this article - sign up in the box at the top right corner of the article or click here.

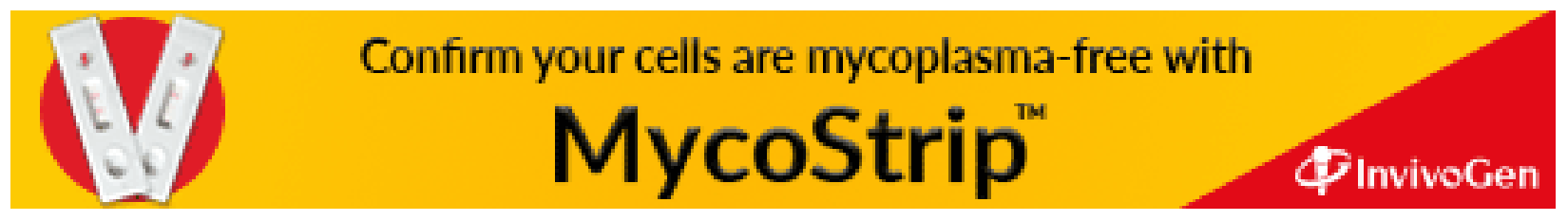

